\section{P80 \\ BSG POLYP SURVEILLANCE GUIDELINES 2020: A SCOPE FOR CHANGE}

Sophie Williams*, Rosemary Barker, Alexander Ribbits, Yooyun Chung, Mehul Patel, Bu'Hussain Hayee, Amyn Haji, Shraddha Gulati, Robert Logan. King's College Hospital, London, UK

\subsection{6/gutjnl-2020-bsgcampus. 155}

Introduction In 2020, the British Society of Gastroenterology (BSG), the Association of Coloproctology of Great Britain and Ireland, and Public Health England revised post-polypectomy surveillance guidelines. Here, we compare colonoscopy findings and planned surveillance dates at our institution, a tertiary referral centre, with novel recommendations in all patients awaiting surveillance colonoscopy following polypectomy.

Methods All patients awaiting surveillance colonoscopy were collated by endoscopy management. Electronic patient records were accessed to obtain latest colonoscopy findings, histology results and follow-up recommendations. Results and recommendations were compared with the 2020 guidelines and an up-to-date recommendation generated.

Results 330 cases were analysed from 2016-2020. According to new guidance, 74 procedures should continue as planned (22.4\%), 221 colonoscopies are not required (66.9\%), 7 should be brought forward (2.1\%) and 17 pushed back (5.2\%). There were 11 cases $(3.3 \%)$ in which the recommendation was not clear. Reasons included missing data and declined procedures.

Of the colonoscopies to continue as planned, 31 had high risk findings, 12 had low risk findings, 26 were normal and 4 had other findings (eg inflammatory bowel disease). Of the surveillance colonoscopies no longer required, 14 were high risk patients over 75 years, 178 were low risk and 29 were normal. The majority of planned surveillance colonoscopies not needed were previously scheduled for either 3 or 5 year follow up.

Conclusions We present data from a single tertiary referral centre over a four year period. Application of the novel guidance has led to approximately $70 \%$ of patients avoiding unnecessary colonoscopies and the associated procedural risks. The cost of a colonoscopy at our centre is $£ 622$. We anticipate a potential cost saving of $£ 137,462$ in this cohort. Our data suggest that application of the 2020 polyp surveillance guidelines can provide tangible benefits both financially and for patients.

\section{P81 A COLORECTAL REFERRAL PATHWAY INCORPORATING PRIMARY CARE FAECAL HAEMOGLOBIN TESTING SAFELY AND EFFECTIVELY PRIORITISES INVESTIGATION}

\begin{abstract}
${ }^{1}$ Stephen McSorley, ${ }^{2}$ Paul Witherspoon, ${ }^{5}$ Douglas Rigg, ${ }^{3}$ Paul Burton, ${ }^{4}$ Jack Winter* ${ }^{1}$ University Department of Surgery, Glasgow, UK; ${ }^{2}$ Department of Surgery, Queen Elizabeth University Hospital, Glasgow, UK; ${ }^{3}$ Information Manager, Greater Glasgow and Clyde Health Board, Glasgow, UK; ${ }^{4}$ Department of Gastroenterology, Glasgow Royal Infirmary, Glasgow, UK; ${ }^{5}$ West of Scotland Primary Care Cancer Network, Glasgow, UK
\end{abstract}

\subsection{6/gutjnl-2020-bsgcampus. 156}

Introduction There is increasing interest in using Quantitative Faecal Immunochemical Testing (QFIT) for Haemoglobin as a 'rule out' test for significant colonic disease in symptomatic patients. It has been demonstrated elsewhere in Scotland that incorporating faecal haemoglobin testing into a primary care clinical assessment tool can safely and effectively prioritise referral for colorectal investigation. Here we present results of our own experience in the largest Scottish Health board (population 1.14 million) following adoption of a primary care colorectal referral pathway incorporating QFIT.

Methods A new referral pathway incorporating primary care testing of faecal haemoglobin was incorporated into clinical practice in September 2018, regardless of patient age. Faecal haemoglobin was measured using the HMJack analyser (Kyowo-Medex), with a cut off for a positive test being 10 $\mathrm{ug} / \mathrm{g}$ stool. The need for, and priority of, investigation, was determined according to the faecal haemoglobin concentration along with assessment for other pre-determined 'red flag' symptoms (iron deficiency anaemia, persistent rectal bleeding or daily diarrhoea $>4$ weeks, rectal or abdominal mass). Ascertainment of significant colonic disease was determined after 1 year of follow up by linkage of faecal haemoglobin results to local endoscopy, radiology and pathology databases, and finally verified by linkage to the Scottish Cancer Registry.

Results A minimum of 12 month follow up information is available for 3818 patients who submitted a QFIT sample between September and December 2018. A faecal haemoglobin result was available for 3547 patients, and positivity was $25.3 \%$, with $4.4 \%$ of results being above the maximum quantifiable value ( $>400 \mathrm{ug} / \mathrm{g}$ stool). 1312 patients had undergone colonoscopy. 54 patients were diagnosed with colorectal cancer within 1 year of having faecal haemoglobin analysed. 51/ 54 (94.4\%) patients had a positive QFIT test, and 53/54 patients (98.1\%) had their investigation prioritised based on QFIT result or pre-determined 'red flag' symptoms. Only 1/ 3793 patients developed cancer within one year of an undetectable faecal haemoglobin and in the absence of 'red flag' symptoms. Advanced adenomas were found in $9.2 \%$ vs $2.1 \%$ investigated patients with detectable faecal haemoglobin, and inflammatory bowel disease was diagnosed in $6.2 \%$ vs $1.6 \%$. In one sector of the board, introduction of this pathway has reduced demand for 'direct to test' colonoscopy by $20 \%$, and demand for all luminal gastroenterology and colorectal surgery outpatient activity by $12.4 \%$.

Conclusion Adopting a pathway incorporating faecal immunochemical testing for haemoglobin in primary care as an adjunct to a formalised clinical assessment can safely determine a patient's risk of significant colorectal pathology, particularly colorectal cancer, and help prioritise investigation.

\section{P82 IMPROVING USE OF CALPROTECTIN IN PAEDIATRIC PRIMARY CARE - AN EVIDENCED-BASED ALGORITHMIC APPROACH TO DECISION-MAKING}

${ }^{1}$ George Averill*, ${ }^{1}$ Emily Warren, ${ }^{1}$ Hazel Green, ${ }^{2}$ Adrian Brown, ${ }^{1}$ Rebecca Hopkins, ${ }^{1}$ Anthony Wiskin. ' University Hospitals Bristol, UK; ${ }^{2}$ North Bristol NHS Trust, UK

\subsection{6/gutjnl-2020-bsgcampus.157}

Introduction Faecal calprotectin (FCP) is recommended in UK NICE guidance as a tool in distinguishing inflammatory bowel disease (IBD) from Irritable Bowel Syndrome in adults. Its utility for the same purpose in children is poorly understood and there has been concern in our hospital that a high rate of testing and uncertainty in test interpretation may be driving an increase in avoidable referrals from primary care. We sought to determine whether this assessment was supported by local data and to ascertain whether a paediatric FCP algorithm 